\title{
Novel Design Methodology of Matching Networks based on the use of Symmetry Operators
}

\author{
Alfred Grau Besoli*1, Jordi Romeu ${ }^{2}$, Lluis Jofre ${ }^{2}$, and Franco De \\ Flaviis $^{1}$ \\ ${ }^{1}$ University of California at Irvine, Irvine, CA 92697, USA \\ ${ }^{2}$ Universitat Politecnica de Catalunya, Barcelona, Spain \\ E-mail: agrau@alumni.uci.edu
}

\section{Introduction}

In this paper we present a novel design methodology of matching networks. The functionality of a matching network is two-fold: first to decouple the antennas of a particular antenna array and secondly to match the resultant ports. The benefits of using a matching network are several: the antenna elements of a particularly antenna array can be closely spaced thus producing compact array designs (but at the expenses of narrower bandwidth), and it can be shown that the produced set of radiation patterns/polarization are orthogonal among each other (assuming an ideal NLOS scenario), thus producing maximum diversity gains when employed in MIMO systems. Several methods to design matching networks exist in the literature [1][2]. Most of these designs methods, do not provide a general procedure to derive such networks for any number of antenna elements, topologies or antenna spacing. Instead, the proposed methodology is valid for any number of antenna elements, array topology, and antenna spacing. It is based on the use of the Takagi factorization, which allows us to produce reciprocal lossless designs. To compute this factorization we make use of the Symmetry Operators Theory to simply derive these networks from a physical inspection of the symmetry properties of the antenna array.

\section{Proposed Design Methodology}

Assume an antenna array with $N$ radiating elements. Let us consider the case in which the matching network is reciprocal and lossless. Let $\mathbf{S}^{D N}$ be its scattering matrix that verifies the unitarity and reciprocity conditions [3]. As shown in [4], the sub-block matrices of $\mathbf{S}^{D N}$ may then be expressed as:

$$
\begin{aligned}
& \mathbf{S}_{11}^{D N}=\mathbf{V}_{11}^{D N} \mathbf{D}_{11}^{D N}\left(\mathbf{V}_{11}^{D N}\right)^{T} \\
& \mathbf{S}_{12}^{D N}=\mathbf{V}_{11}^{D N} \mathbf{D}^{D N}\left(\mathbf{V}_{22}^{D N}\right)^{T} \\
& \mathbf{S}_{21}^{D N}=\mathbf{V}_{22}^{D N} \mathbf{D}^{D N}\left(\mathbf{V}_{11}^{D N}\right)^{T} \\
& \mathbf{S}_{22}^{D N}=\mathbf{V}_{22}^{D N} \mathbf{D}_{22}^{D N}\left(\mathbf{V}_{22}^{D N}\right)^{T}
\end{aligned}
$$

where $\mathbf{V}_{i i}^{D N}$ are unitary matrices, and $\mathbf{D}_{i i}^{D N}$ and $\mathbf{D}^{D N}$ diagonal matrices. According to [5], the aforementioned matching network can be decomposed into three inner sub-networks. Within this decomposition, the end-ones are all-passes subnetworks (also named decoupling networks) and the middle one is a bank of $N$ uncoupled 2-port ideal transformers (also named matching network). Let us define by $\mathbf{S}^{D L} \in$ $\mathcal{C}^{2 N \times 2 N}$ and $\mathbf{S}^{D R} \in \mathcal{C}^{2 N \times 2 N}$ the scattering matrices corresponding to the left side and right side decoupling networks. Let $\mathbf{S}^{M} \in \mathcal{C}^{2 N \times 2 N}$ be the scattering matrix of 
the matching network. These matrices can be expressed as a function of the terms in Eqs. (1) to (4), as follows

$$
\mathbf{S}^{D L}=\left(\begin{array}{cc}
\mathbf{0} & \mathbf{V}_{11}^{D N} \\
\left(\mathbf{V}_{11}^{D N}\right)^{\dagger} & \mathbf{0}
\end{array}\right) \mathbf{S}^{D R}=\left(\begin{array}{cc}
\mathbf{0} & \left(\mathbf{V}_{22}^{D N}\right)^{\dagger} \\
\mathbf{V}_{22}^{D N} & \mathbf{0}
\end{array}\right) \mathbf{S}^{M}=\left(\begin{array}{ll}
\mathbf{D}_{11}^{D N} & \mathbf{D}^{D N} \\
\mathbf{D}^{D N} & \mathbf{D}_{22}^{D N}
\end{array}\right)
$$

Let us assume now that $\Gamma^{1}$ is the scattering matrix of a reciprocal lossless antenna array. To ensure that the matching efficiency at the inputs of the matching network is maximized it is sufficient that $\mathbf{S}_{11}^{D N}=\left(\boldsymbol{\Gamma}^{1}\right)^{H}$, which happens to be independent of the chosen value for $\mathbf{S}_{22}^{D N}$. Therefore, the right-side decoupling sub-network becomes unnecessary in our case, and thus its scattering matrix can be chosen to be the identity matrix. In the case $\Gamma^{1}$ is a complex symmetrical matrix [6], the Takagi Singular Value Decomposition (TSVD) [6] can be used to factorize it. Therefore, $\Gamma^{1}$ can be decomposed as

$$
\Gamma^{1}=\mathbf{V}^{R} \mathbf{D}^{R}\left(\mathbf{V}^{R}\right)^{\dagger}
$$

where $\mathbf{V}^{R}$ is a unitary matrix and $\mathbf{D}^{R}$ is a complex diagonal matrix with unitmagnitude entries. Finally, having in mind that the design condition is $\mathbf{S}_{11}^{D N}=$ $\left(\boldsymbol{\Gamma}^{1}\right)^{H}$, we can equate Eq. (1) and the Hermitian of Eq. (6) to readily obtain

$$
\begin{gathered}
\mathbf{V}_{11}^{M R}=\left(\mathbf{V}^{R}\right)^{*} \\
\mathbf{D}_{11}^{M R}=\left(\mathbf{D}^{R}\right)^{H}
\end{gathered}
$$

The last step of the presented methodology is to factorize $\Gamma^{1}$. Symmetry operators [7] are mathematical tools that can be used to describe the symmetry characteristics of antenna arrays, or as in our case, to compute the factorization given in Eq. (6), in a very intuitive manner. For example, a symmetrical antenna array is characterized by the fact that it is left unchanged by a symmetry operation. That is, in the case of a $2 \times 1$ linear antenna array antenna configuration, for example, the symmetry operation is a rotation of the structure by $180^{\circ}$ about the symmetry axis, as shown in Fig. 1 (a). This operation turns the figure back into itself. The antenna array is said to be invariant under this symmetry operation. The group of symmetry operations of an antenna array is based on reflections or rotations which transform the array into itself, and are normally represented by matrices $\mathbf{F}$ to denote reflection with respect to a plane, $\mathbf{R}$ to denote rotation with respect to an axis, and $\mathbf{P}$ to refer to a reflection in the origin. In can then be shown that the operators $\mathbf{F}$ and $\boldsymbol{\Gamma}^{1}$ commute. The same development applies for the $\mathbf{R}$ and $\mathbf{P}$ symmetry operators. One now recalls the fact that two operators commute if, and only if, the operators possess a common set of eigenvectors (common invariant subspaces corresponding to different eigenvalues of $\mathbf{F}$ (or $\mathbf{R}$, or $\mathbf{P}$ ). In other words, there exist a basis which simultaneously completely reduces $\mathbf{F}$ (or $\mathbf{R}$, or $\mathbf{P}$ ) and $\boldsymbol{\Gamma}^{1}$. A transformation $\mathbf{V}^{R}$ which completely reduces $\Gamma^{1}$, is then deduced from the simple, known, symmetry operators. That is, it is possible to have $\boldsymbol{\Gamma}^{1}=\mathbf{V}^{R} \mathbf{D}^{R}\left(\mathbf{V}^{R}\right)^{-1}$ where $\mathbf{V}^{R}$ and $\mathbf{D}^{R}$ have been obtained from the well known eigenvalue decomposition of $\mathbf{F}$ (or $\mathbf{R}$, or $\mathbf{P}$ ). It is important that the final result is such that $\mathbf{V}^{R}$ is composed of pure real and orthogonal vectors. In that case, $\left(\mathbf{V}^{R}\right)^{-1}=\left(\mathbf{V}^{R}\right)^{\dagger}$, and therefore we have a reciprocal design for the matching network, that verifies $\Gamma^{1}=\mathbf{V}^{R} \mathbf{D}^{R}\left(\mathbf{V}^{R}\right)^{-1}=\mathbf{V}^{R} \mathbf{D}^{R}\left(\mathbf{V}^{R}\right)^{\dagger}$. In general, it 
is possible to choose eigenvectors of $\boldsymbol{\Gamma}^{1}$ that are real, by doing linear combinations of the original ones found from the eigenvalue decomposition of the symmetry operators associated with the antenna array.

\section{Example of Microstrip Decoupling Network}

Two illustrate the abovementioned procedure we design a matching network for the simplest possible antenna array, a 2-element linear array. A 2-element antenna array has a scattering matrix, $\boldsymbol{\Gamma}^{1}$, of the form

$$
\Gamma^{1}=\left(\begin{array}{ll}
\Gamma_{11}^{1} & \Gamma_{12}^{1} \\
\Gamma_{12}^{1} & \Gamma_{11}^{1}
\end{array}\right)
$$

For this particular antenna array there exist only one independent symmetry operator $\mathbf{F}$, given by $\mathbf{F}=\left(\begin{array}{ll}0 & 1 \\ 1 & 0\end{array}\right)$ which can be obtained from physical inspection in Fig. 1 (a), as suggested in [7]. The associated eigenvalues of the operator $\mathbf{F}$ are $\lambda_{1}=1$ and $\lambda_{2}=-1$. The invariant subspaces are therefore non-degenerated, because all the eigenvalues are different, and the eigenvectors are given by

$$
\mathbf{v}_{1}=\frac{1}{\sqrt{2}}\left(\begin{array}{l}
1 \\
1
\end{array}\right), \mathbf{v}_{2}=\frac{1}{\sqrt{2}}\left(\begin{array}{c}
1 \\
-1
\end{array}\right)
$$

Therefore the transformation matrix $\mathbf{V}^{R}$ can be built by placing the previous eigenvectors in its columns. In this case, $\boldsymbol{\Gamma}^{1}$ can be decomposed into $\boldsymbol{\Gamma}^{1}=\mathbf{V}^{R} \mathbf{D}^{R}\left(\mathbf{V}^{R}\right)^{\dagger}$ where $\mathbf{D}^{R}$ can be written as $\mathbf{D}^{R}=\left(\begin{array}{cc}\Gamma_{11}^{1}+\Gamma_{12}^{1} & 0 \\ 0 & \Gamma_{11}^{1}-\Gamma_{12}^{1}\end{array}\right)$ which is the desired solution. Finally, $\mathbf{V}^{R}$ and $\mathbf{D}^{R}$ can be used as in the previous section to synthesize the matching network. In particular, the scattering matrix of the decoupling network, $\mathbf{S}^{D L}$ is given by

$$
\mathbf{S}^{D L}=\frac{1}{\sqrt{2}}\left(\begin{array}{cccc}
0 & 0 & 1 & 1 \\
0 & 0 & 1 & -1 \\
1 & 1 & 0 & 0 \\
1 & -1 & 0 & 0
\end{array}\right)
$$

which can be readily identified as an $180^{\circ}$-hybrid. A same procedure can be followed for more complex antenna array configurations. These more advanced examples will be presented at the conference. Simulated results are presented in Fig. 1. In part (b), it is shown the scattering paramters for the 2-element antenna array for an antenna separation of $0.14 \lambda$ and $0.5 \lambda$. Finally, part (c) shows the resultant simulated scattering parameters at the inputs of the matching network when it is connected to the antenna array, for the case of an antenna separation of $0.14 \lambda$. Notice that for an antenna separation of $0.14 \lambda$, the original coupling values $\left(S_{i j}\right)$ are around $-6 \mathrm{~dB}$, while when the matching network is used, the coupling values are below $-28 \mathrm{~dB}$. The matching parameters $\left(S_{i i}\right)$ also improve from $-10 \mathrm{~dB}$ down to $-20 \mathrm{~dB}$. On the other hand, the operational bandwidth is narrowed. Measurements results will be also presented at the conference. 


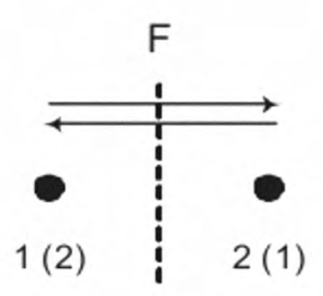

(a)

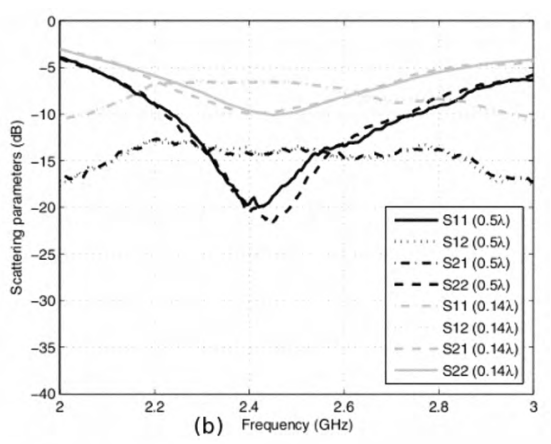

(b) Frequency $(\mathrm{GHz}$

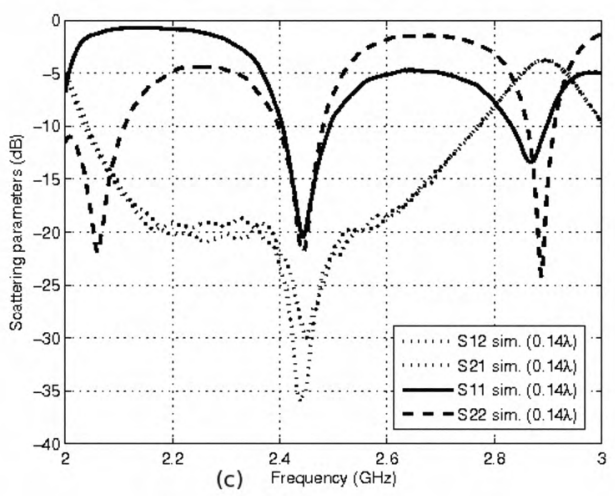

Figure 1: Set of figures described within the paper.

\section{References}

[1] J. B. Andersen and H. H. Rasmussen, "Decoupling and descattering networks for antennas," Antennas and Propagation, IEEE Transactions on, pp. 841-846, Nov 1976.

[2] S. Dossche, S. Blanch, and J. Romeu, "Three different ways to decorrelate two closely spaced monopoles for MIMO applications," Wireless Communications and Applied Computational Electromagnetics, 2005. IEEE/ACES International Conference on, pp. 849-852, Apr 2005.

[3] D. M. Pozar, Microwave Engineering, 2nd ed. John Wiley and Sons Inc., 1998.

[4] J. W. Wallace and M. A. Jensen, "Termination-dependent diversity performance of coupled antennas: Network theory analysis," IEEE Transactions on antennas and propagation, vol. 52, no. 1, pp. 98-105, Jan 2004.

[5] D. C. Youla, "Weissfloch equivalents for lossless 2n-ports," IEEE TRANSACTIONS ON CIRCUIT THEORY,, pp. 193-199, Sep 1960.

[6] R. A. Horn and C. R. Johnson, Matrix Analysis, 1st ed. Cambridge University Press, 1985.

[7] E. R. Beringer, N. Marcuvitz, C. G. Montgomery, R. H. Dicke, and E. M. Purcell, Principles of Microwave Circuits, Radiation Laboratory Series, 1st ed. McGraw Hill Book Companny, Inc., 1945. 